\title{
The IT Dilemma and the Unified Computing Framework
}

\author{
Edwin Paalvast \\ Vice President Service Sales Europe \\ Cisco Systems \\ Haarlerbergweg 13-19, $1101 \mathrm{CH}$ Amsterdam \\ The Netherlands
}

\begin{abstract}
One of the main challenges of the IT industry is the portion of the IT budget spent on maintaining the IT systems. For most companies this is around $80-85 \%$ of the total budget. This leaves very little room for new functionality and innovation. One of the ways to save money is to make more effective use of the underlying hardware like disks and processors. With the freed up budget the real IT issue can be addressed: the cost of application maintenance. With the use of publish-subscribe and agent models the changes in policies and business models can be supported more quickly, but it requires the right underlying infrastructure. I will discuss a Unified Computing framework that will enable these savings and will have the required capabilities to support model based programming.
\end{abstract}

